\title{
Crystal structure of a plant leucine rich repeat protein with two island domains
}

\author{
SONG Wen, HAN ZhiFu, SUN YaDong \& CHAI JiJie* \\ School of Life Sciences, Tsinghua-Peking Center for Life Sciences, Tsinghua University, Beijing 100084, China
}

Received October 15, 2013; accepted November 20, 2013; published online December 23, 2013

\begin{abstract}
Leucine rich repeat (LRR) domain, characterized by a repetitive sequence pattern rich in leucine residues, is a universal protein-protein interaction motif present in all life forms. LRR repeats interrupted by sequences of 30-70 residues (termed island domain, ID) have been found in some plant LRR receptor-like kinases (RLKs) and animal Toll-like receptors (TLR7-9). Recent studies provide insight into how a single ID is structurally integrated into an LRR protein. However, structural information on an LRR protein with two IDs is lacking. The receptor-like protein kinase 2 (RPK2) is an LRR-RLK and has important roles in controlling plant growth and development by perception and transduction of hormone signal. Here we present the crystal structure of the extracellular LRR domain of RPK2 (RPK2-LRR) containing two IDs from Arabidopsis. The structure reveals that both of the IDs are helical and located at the central region of the single RPK2-LRR solenoid. One of them binds to the inner surface of the solenoid, whereas the other one mainly interacts with the lateral side. Unexpectedly, a long loop immediately following the N-terminal capping domain of RPK2-LRR is presented toward and sandwiched between the two IDs, further stabilizing their embedding to the LRR solenoid. A potential ligand binding site formed by the two IDs and the solenoid is located at the C-terminal side of RPK2-LRR. The structural information of RPK2-LRR broadens our understanding toward the large family of LRR proteins and provides insight into RPK2-mediated signaling.
\end{abstract}

leucine rich repeat, receptor-like kinases, receptor-like protein kinase 2

Citation: $\quad$ Song W, Han ZF, Sun YD, Chai JJ. Crystal structure of a plant leucine rich repeat protein with two island domains. Sci China Life Sci, 2014, 57: 137-144, doi: 10.1007/s11427-013-4586-x

The leucine rich repeat (LRR) is a versatile protein motif ( 20-30 amino acids) found in a variety of proteins in all life forms. Tandem arrays of two or more repeats in an LRR domain form a structure similar to a horse-shoe shaped solenoid mainly based on structural studies of animal TLRs [1,2]. However, recent structural investigations [3-6] of plant LRR proteins have challenged this "dogma". These studies reveal that plant LRR containing proteins adopt a superhelical rather than a planar horse-shoe shaped structure. Largely by acting as scaffolds for protein-protein or protein-ligand interactions, LRR domains participate in many biological processes [1,2]. This is well exemplified by the

*Corresponding author (email: chaijj@mail.tsinghua.edu.cn) large family of plant LRR receptor-like kinases (RLKs) that recognize highly diversified ligands, ranging from proteins, sugars to hormones [7]. Nearly all of the known typical LRR structures comprise an N-terminal (LRRNT) cap and some of them have a C-terminal (LRRCT) cap as well, shielding the two ends of the conserved central LRR segment [1,2]. As indicated by its name, an LRR domain is characterized by a repetitive sequence pattern rich in leucine residues. However, LRR proteins including some plant LRR-RLKs and mammalian Toll-like receptors (TLR7-9), in which LRR repeats are interrupted by sequences of 30-70 residues termed island domain or non-LRR region, have been found [8]. BRI1 contains one ID and its interrupted LRRs pack continuously [5,6], forming a single so- 
lenoid structure as observed in all the other LRR proteins with structures known. However, continuous packing of LRRs is disrupted in the ID-containing of TMK1-LRR, resulting in formation of two solenoid structures within a single LRR protein [4].

Plants produce new tissues and organs primarily postembryonically from pluripotent stem cells in the shoot apical meristems (SAM) and root apical meristems (RAM). Through cell-to-cell communication, both meristems can maintain proper meristematic activities and meristem organization and coordinate new organ formation [9-11]. In SAM, an essential regulatory mechanism for meristem organization is a negative feedback loop between WUSCHEL (WUS) and CLAVATA (CLV). WUS expression, which is repressed at the transcriptional level by a small signaling peptide encoded by CLAVATA3 (CLV3) secreted from stem cells, promotes stem cell fate [9-14]. Genetic studies show that CLV1, CLV2-CRN/SOL2 and RPK2 receptor complexes function for CLV3 recognition in Arabidopsis [13-15]. The three major receptor complexes are likely to mediate three signaling pathways, mainly in parallel but with potential crosstalk, to regulate the SAM homeostasis [14]. Emerging evidence shows that parallel signaling pathways involving CLV1, CLV2, and RPK2 are important for nematode parasitism [16,17]. Additionally, RPK2 is also critical for other biological processes. For example, RPK2 and its homolog RPK1 (76\% identity in their kinase domains) are redundantly required for Arabidopsis embryonic pattern formation $[18,19]$. Recent studies found that RPK2 is a critical factor controlling anther development in Arabidopsis thaliana probably via CLV/WUS-mediated growth control as suggested $[9,19,20]$.

\section{Materials and methods}

\subsection{Protein purification}

The LRR domain of RPK2 (residues 44-811, RPK2-LRR) from Arabidopsis with an engineered C-terminal $6 \times$ His tag was generated by standard PCR-based cloning strategy and its identity was confirmed by sequencing. The protein was expressed in high five cells at $22^{\circ} \mathrm{C}$ using the vector pFastBac 1 (Invitrogen) with a modified N-terminal Hemolin peptide. One litre of cells $\left(1.5 \times 10^{6}\right.$ cells $\left.\mathrm{mL}^{-1}\right)$ was infected with $20 \mathrm{~mL}$ baculovirus and the protein was harvested from the media after $48 \mathrm{~h}$. The protein was purified using Ni-NTA (Novagen) and size-exclusion chromatography (Hiload 200, GE Healthcare, Shanghai, China) in buffer (10 mmol L ${ }^{-1}$ Bis-Tris (pH 6.0) and $100 \mathrm{mmol} \mathrm{L}^{-1} \mathrm{NaCl}$ ). Samples from relevant fractions were applied to SDS-PAGE and visualized by Coomassie blue staining. Protein purification was performed at $4^{\circ} \mathrm{C}$. For crystallization of RPK2-LRR, the purified protein was concentrated to about $10.0 \mathrm{mg}$ $\mathrm{mL}^{-1}$ in buffer containing $10 \mathrm{mmol} \mathrm{L}^{-1}$ Bis-Tris $(\mathrm{pH} 6.0$ ) and $100 \mathrm{mmol} \mathrm{L}^{-1} \mathrm{NaCl}$.

\subsection{Crystallization, data collection and structural de- termination}

Crystals of RPK2-LRR were generated by the hanging-drop vapor-diffusion method. The drops were set up with $1 \mu \mathrm{L}$ protein plus $1 \mu \mathrm{L}$ reservoir solution at $18^{\circ} \mathrm{C}$. Diffraction quality crystals of the RPK2-LRR were obtained at buffer containing $0.1 \mathrm{~mol} \mathrm{~L}^{-1}$ Hepes sodium ( $\left.\mathrm{pH} 7.0\right), 0.1 \mathrm{~mol} \mathrm{~L}^{-1}$ magnesium chloride hexahydrate, $0.005 \mathrm{~mol} \mathrm{~L}^{-1}$ nickel(II) chloride hexahydrate, $15 \%(\mathrm{w} / \mathrm{v})$ polyethylene glycol (PEG) 3350. All the diffraction data sets were collected at the Shanghai Synchrotron Radiation Facility (SSRF) at beam line BL17U1 using a CCD detector. The data were processed using HKL2000 [22]. The crystal structures of RPK2-LRR were determined by molecular replacement (MR) with PHASER [23] using the structure of BRI1LRR (PDB code: 3RGZ) as a search model. The model from MR was built with the program COOT [24] and subsequently subjected to refinement by the program PHENIX [25]. The structure of the RPK2-LRR was finally refined to a resolution of $2.3 \AA$ with $R_{\text {work }}=21.2 \%$ and $R_{\text {free }}=25.8 \%$. All the structure figures were prepared using PYMOL [26].

\subsection{Gel filtration analysis}

The RPK2-LRR proteins purified as described earlier were subjected to a gel filtration analysis (Hiload 200; GE Healthcare, Shanghai, China). Buffer containing $10 \mathrm{mmol}$ $\mathrm{L}^{-1}$ Bis-Tris ( $\mathrm{pH}$ 6.0) and $100 \mathrm{mmol} \mathrm{L}^{-1} \mathrm{NaCl}$ was used for Hiload200. Samples from relevant fractions were applied to SDS-PAGE and visualized by Coomassie blue staining.

\section{Results and discussion}

\subsection{RPK2-LRR is a monomer in crystals and solution}

Wild type RPK2-LRR (residues 44-811; Figure 1A) protein with a C-terminal $6 \times$ His tag was expressed in insect cells and purified to homogeneity for crystallization. The crystals of RPK2-LRR protein belonged to space group $\mathrm{P} 22_{1} 2_{1} 2_{1}$, with unit-cell parameters $a=72.87 \AA, b=101.14 \AA, c=134.30 \AA$ and $\alpha=\beta=\gamma=90^{\circ}$, and one RPK2-LRR molecule per asymmetric unit. The structure of RPK2-LRR was solved using molecular replacement (Table 1). The finally refined structure contained residues 45-67, 73-481 and 484-747 with excellent stereochemistry. All the remaining residues were not well defined by electron density presumably because they were disordered in solution.

None of the dimeric packing between two RPK2-LRR molecules related by crystallographic symmetry can form a dimer, indicating that RPK2-LRR is monomeric in crystals. To further support the structural observation, we quantified the molecular weight of the RPK2-LRR protein in solution 


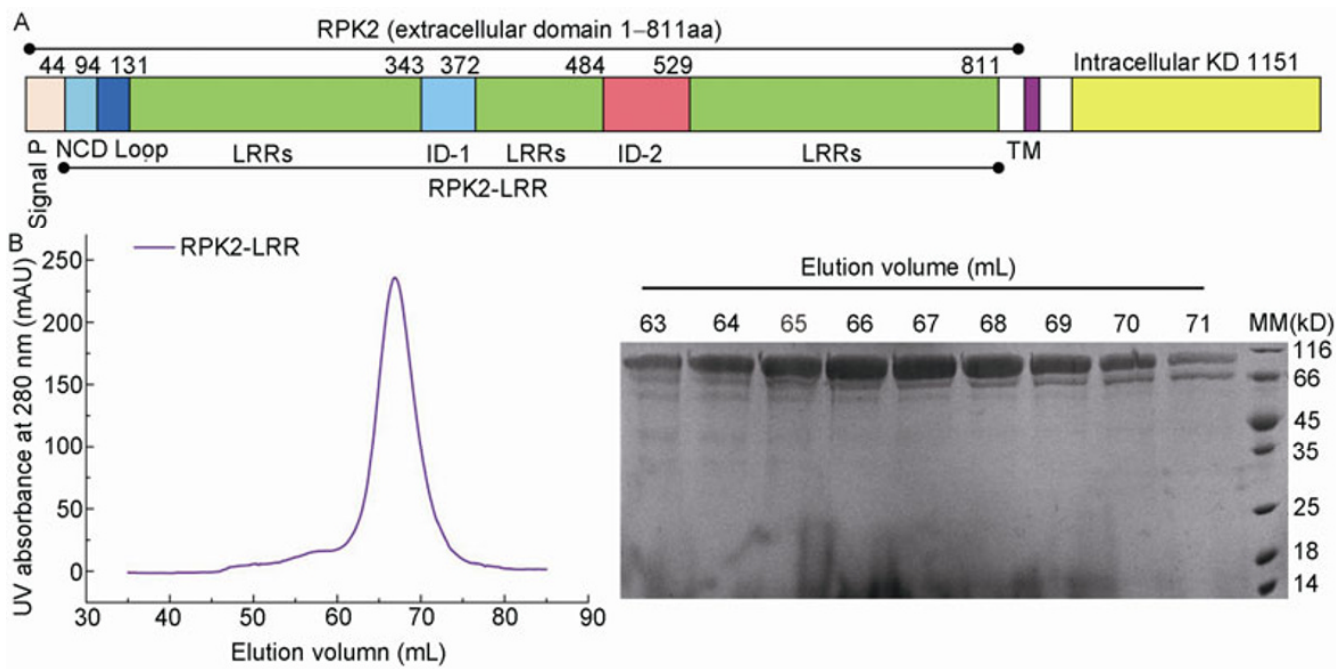

Figure 1 RPK2-LRR is monomeric in solution. A, Structural organization of RPK2 from Arabidopsis. The numbers indicate boundaries of domains. NCD, N-terminal capping domain; LRRs, leucine-rich repeats; ID-1, island domain 1; TM, transmembrane segment; KD, kinase domain. B, Left panel: The gel filtration chromatogram of the RPK2-LRR protein. The vertical and horizontal axes represent ultraviolet absorbance ( $\lambda=280 \mathrm{~nm})$ and elution volume (mL), respectively. The elution volume of RPK2-LRR was $67.0 \mathrm{~mL}$, corresponding to a molecular weight of $\sim 100 \mathrm{kD}$. Right panel: Coomassie blue staining of the peak fraction SDS-PAGE. MM, molecular weight marker.

Table 1 Data collection and refinement statistics

\begin{tabular}{|c|c|}
\hline \multicolumn{2}{|c|}{ RPK2-LRR } \\
\hline Data collection statistics & \\
\hline Beam Line & Beam line BL17U1, SSRF \\
\hline Space Group & $\mathrm{P} 2{ }_{1} 2{ }_{1}{ }_{1}$ \\
\hline Wavelength ( $\mathrm{A})$ & 1.0001 \\
\hline Number of reflections & 44495 (2036) \\
\hline \multicolumn{2}{|l|}{ Cell dimensions } \\
\hline$a, b, c(\AA)$ & $72.869,101.136,134.297$ \\
\hline$\alpha, \beta, \gamma\left(^{\circ}\right)$ & $90.00,90.00,90.00$ \\
\hline Resolution ( $\mathrm{A})$ & $2.3(2.34-2.3)$ \\
\hline$R_{\text {sym }}(\%)$ & $7.6(73.5)$ \\
\hline$I / \sigma(I)$ & 31.7 (1.6) \\
\hline Completeness $(\%)$ & $98.7(91.0)$ \\
\hline Redundancy & $5.0(4.1)$ \\
\hline \multicolumn{2}{|l|}{ Refinement Statistics } \\
\hline Resolution $(\AA)$ & $50.0 \quad 2.3(2.35-2.3)$ \\
\hline Number of reflections & $44411(2493)$ \\
\hline$R_{\text {work }} / R_{\text {free }}(\%)$ & $21.2 / 25.8(29.8 / 34.7)$ \\
\hline $\begin{array}{l}\text { Number of molecules/ } \\
\text { asymmetric unit } \\
\text { r.m.s deviations }\end{array}$ & 1 \\
\hline Bond lengths ( $\mathrm{A})$ & 0.008 \\
\hline Bond angles $\left(^{\circ}\right)$ & 1.462 \\
\hline \multicolumn{2}{|l|}{$\begin{array}{l}\text { Ramachandran (\%) } \\
\text { (from PHENIX) }\end{array}$} \\
\hline Preferred region & 95.63 \\
\hline Allowed region & 4.37 \\
\hline Outliers & 0.00 \\
\hline
\end{tabular}

a) Highest resolution shell is shown in parenthesis.

using a gel filtration assay. Fully supporting the structural observation, the RPK2-LRR protein was eluted at the position of a molecular weight of $\sim 100 \mathrm{kD}$ (Figure 1B), indicating that the RPK2-LRR protein was also monomeric in solution. A previous study [14] suggested that RPK2 formed homo-oligomers when over-expressed in N. benthamiana. A number of factors may be responsible for the discrepancy. The ectodomain of RPK2 rather than full length RPK2 was used for our structural and biochemical assays. Thus, the observed homo-oligomerization of RPK2 might result from its intracellular kinase domain. Similar observations have been made for BRI1LRR [5,6]. While the LRR domain of BRI1 is monomeric as in solution or crystals, the full length BRI1 has been shown to be homo-oligmerized independent of its ligands [27]. Other component(s) from the plant cells used might also contribute to the homo-oligomerization of RPK2. While less likely, it is also possible that the assay used in our study was not sensitive enough to detect a homo-oligomer of RPK2.

\subsection{Overall structure of RPK2-LRR and comparison with other structures of plant LRR proteins}

As predicted from the sequence, the two IDs of RPK2, residues 343-372 and 484-529, form two independent domains that are not integrated into the RPK2-LRR solenoid structure. The existence of the extra two domains does not disrupt continuous packing of the 22 LRRs, which assemble into a super-helical structure with an overall rotation angle about the central axis being approximately $360^{\circ}$ (Figure 2A). Compared to the structure of the LRR-RLK BRI1 (Figure 2B) [5,6] or BRL1 [3], RPK2-LRR exhibits a more elongated structure and has a smaller size in the diameter of the super-helix, though the latter has fewer LRRs than the other two (with 25 LRRs). One reason for this may be that interactions with the two IDs and the long loop from the $\mathrm{N}$-terminal capping domain distort the super-helical structure of RPK2-LRR. The different structure of RPK2-LRR from BRI1 and BRL1 may also result from the fact that 
LRR14-16 lack the plant specific sequence GxL/i/vP (x stands for any amino acid), which was proposed [5,6] to contribute to a super-helical structure of a plant LRR protein. A salient feature of the RPK2-LRR structure is that it contains three non-canonical LRR repeats (LRR14-16). Similar LRRs also exist in the F-box containing protein CORONATINE INSENSITIVE 1 (COI1) from Arabidopsis that does not contain the plant specific sequence and adopts a non-helical structure [28]. LRR14 incorporates the 2nd ID (ID-2) and contains an unusual hydrophilic residue His534 at the hydrophobic side of the inner solenoid surface. The polarity of this residue is neutralized by hydrogen bonding interaction with the carbonyl oxygen of Met461 and the hydroxyl group of Tyr561 (Figure 2A). These interactions might contribute to the continuous packing of LRR12 against LRR13. The disulfide bond Cys582-Cys609 may have a role in stabilizing LRR16 and LRR17. Despite the striking differences between RPK2-LRR and other LRRRLKs, their N-terminal capping domains display a conserved structure stabilized by a pair of invariable disulfide bond (not shown). Similar to the structures of other RLK-LRRs, several potential glycosylation sites (not shown) as defined by sufficient electron density were found in
RPK2-LRR, but assignment of functions to these glycosylation sites awaits further investigations. The cysteine residues that form disulfide bonds are highly conserved among RPK2s from different plant species (Figure 3 ).

\subsection{Interactions of the RPK2-LRR solenoid with the two IDs}

The 1st ID (ID-1, residues 343-372) contains three short $\alpha$-helices that pack against one lateral side of the RPK2-LRR solenoid via both hydrogen bonds and hydrophobic interactions (Figure 4A). Center to the interaction network are the hydrogen bonds formed between Arg355 and the carbonyl oxygen atoms of Asn247, Gly271 and Asp359. Hydrogen bonds also come from Arg319 that establishes polar interactions with its neighboring three residues (Asn343, Tyr345 and Met369) from the ID-1. The aromatic residues Trp248 and Phe 273 from the periphery of the interface uphold the ID-1 by stacking against the central region of its main chain.

ID-2 (residues 484-529) is mainly helical. The longest $\alpha$-helix from this domain nearly parallels with the central axis of the super-helical solenoid and interacts with the in-
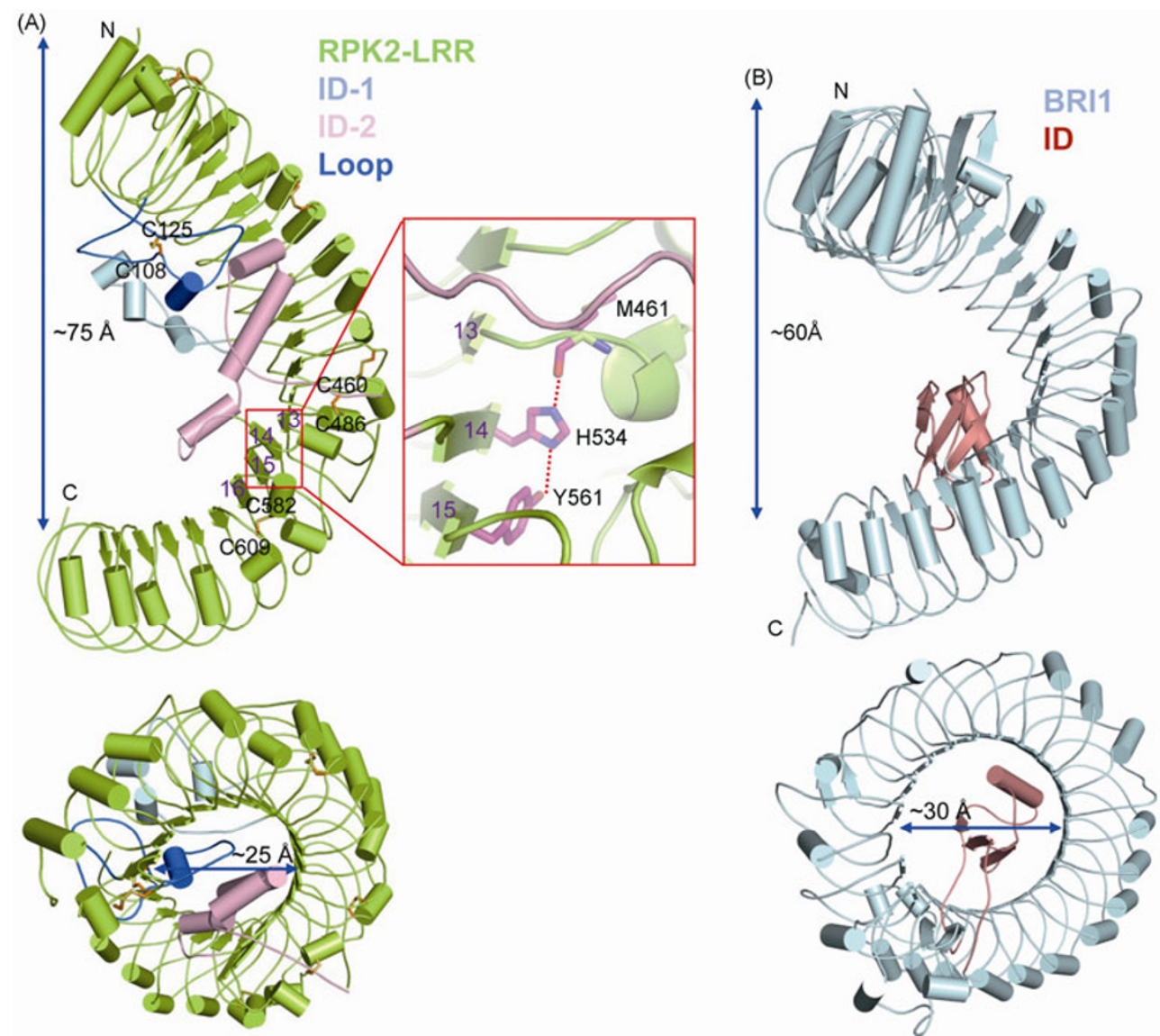

Figure 2 RPK2-LRR adopts a superhelical structure comparable to BRI1-LRR. (A), Overall structures of RPK2-LRR in two different orientations. Disulfide bonds are shown in stick and colored in orange. The purple numbers indicate the positions of LRRs. "N" and "C" indicate the N- and C-terminus, respectively. Dashed red lines indicate hydrogen bonds. (B), Overall structures of BRI1-LRR in two different orientations. 


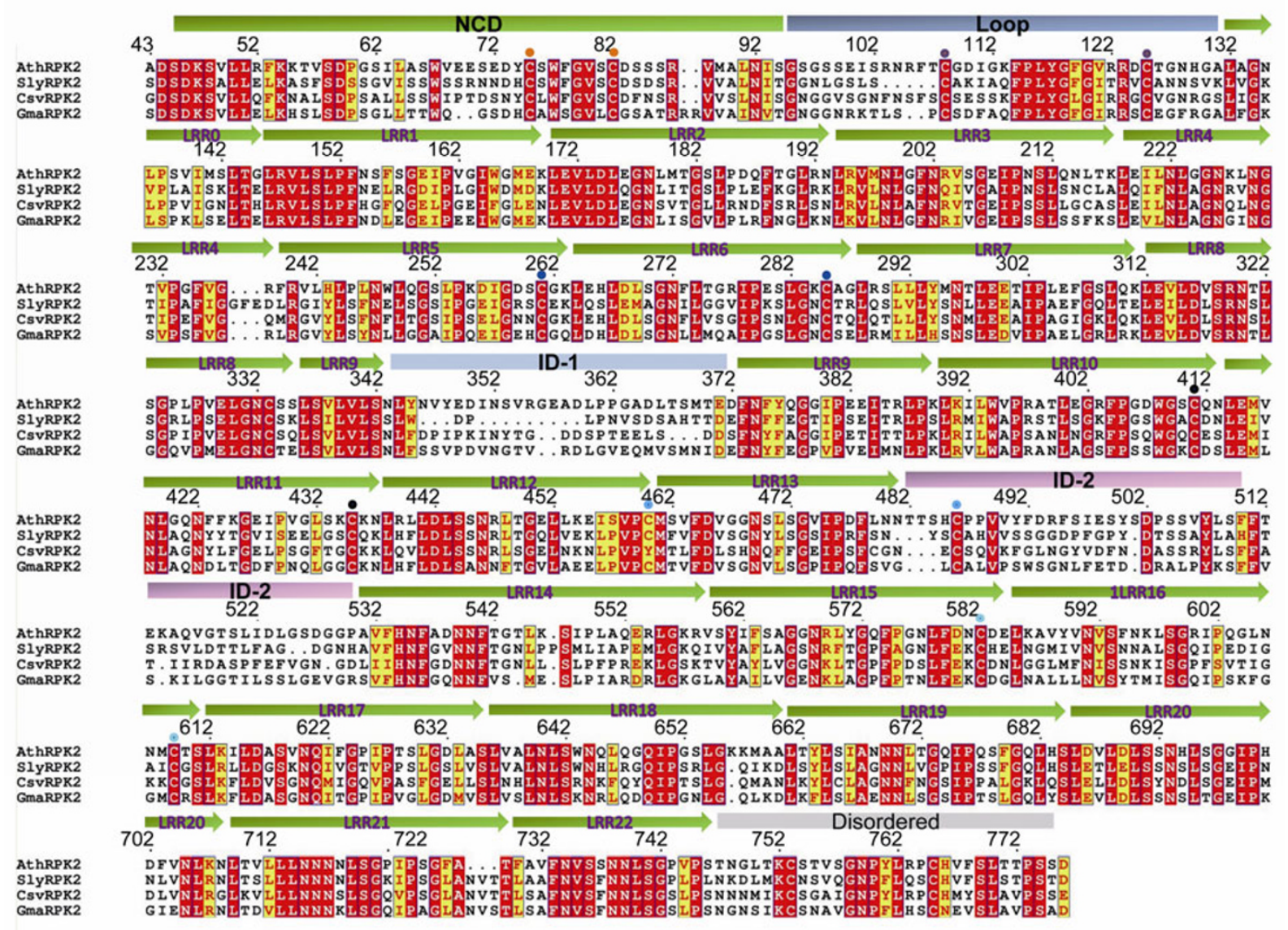

Figure 3 Sequence alignment of RPK2 ectodomain among different species. Conserved and similar residues are highlighted with red and yellow grounds, respectively. Two cysteine residues that form a disulfide bond are indicated by the same color of solid circles on the top. LRR0 is half of an LRR. "NCD" represents the N-terminal capping domain. "Ath", "Sly", "Csv", and "Gma" represent Arabidopsis thaliana, Solanum lycopersicum (tomato), Cucumis sativus (cucumber) and Glycine max (soybean), respectively.

ner surface of the solenoid, spanning LRR8-LRR12 (Figure 4B). The interaction is predominantly hydrophobic (Figure 4B). Five residues (Val314, Val338, Ile392, Met416 and Arg439), one from each LRR of LRR8-12, are positioned linearly to pack against one side of the long $\alpha$-helix. The disulfide bond Cys460-Cys486 (Figure 2A) appears also critical for stabilization of the conformation of the ID. One side of the $\alpha$-helix also packs against those residues from ID-1, thus mediating interactions of the two IDs (Figure 4C). Specifically, Phe510 establishes hydrophobic contacts with Leu345 and Tyr347, whereas Tyr347 in turn interacts with the hydrophobic portion of Lys514 and Ala515.

\subsection{A long loop from the N-terminal side stabilizes the two IDs}

Previous structural studies showed that LRR-RLKs have a conserved $\mathrm{N}$-terminal capping domain consisting of an anti-parallel $\beta$-sheet and three $\alpha$-helices. While some parts were not well defined by electron density, the N-terminal capping domain of RPK2-LRR largely has a structure similar to those of other LRR-RLKs. The capping domain is structurally followed by an LRR (Figure $2 \mathrm{~A}$ ), but at the sequence level by more than 30 amino acids (residues 94-129) predicted not to form an LRR repeat (Figure 3). In the structure, these non-LRR encoding residues form a long loop that covers one lateral side of the solenoid, adjacent to ID-1 (Figure 5A). The disulfide bond Cys108-Cys125 (Figure $2 \mathrm{~A}$ ) likely has a critical role in organizing the conformation of the long loop. At one side of the disulfide bond, the long loop mainly interacts with a lateral side of the solenoid via hydrophobic contacts (Figure 5A). At the other side of the disulfide bond, part of the loop is sandwiched between the two IDs and the remaining part wedges between ID-2 and the inner surface of the solenoid. In addition to hydrogen bonding with the carbonyl oxygen of Pro115, Tyr500 forms $\pi-\pi$ interaction with Arg122. Gly120 forms a hydrogen bond with Tyr500 from ID-2, whereas Phe119 makes hydrophobic contacts with those residues from the inner surface of the solenoid. Leu116 forms simultaneous interactions with both of the two IDs by making hydrophobic contacts with Val347 from ID-1 and Tyr507 from ID-2. The interactions of the loop with ID-1 are also mediated by hydrophobic contacts (Figure 5B). The extensive interac- 

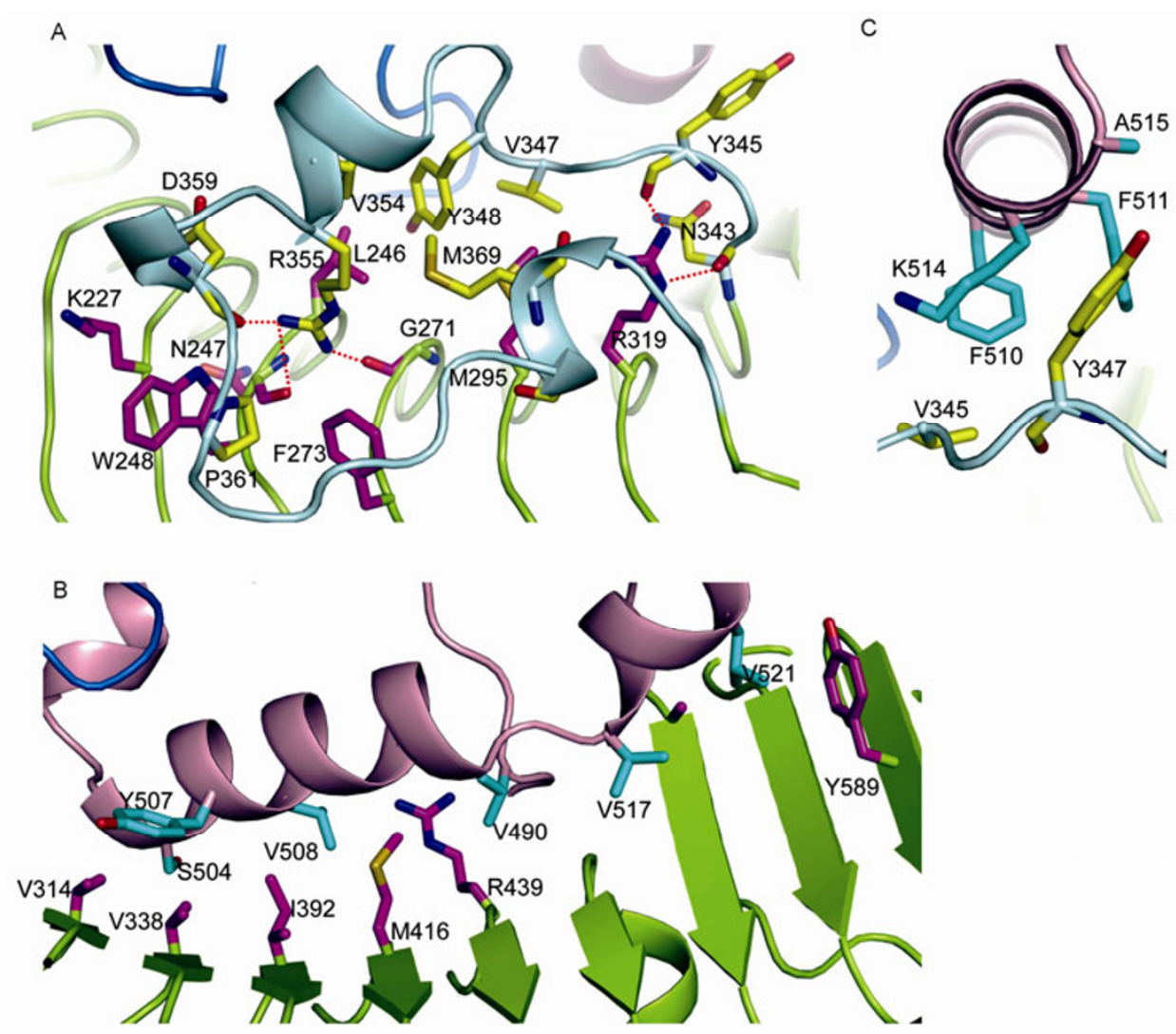

Figure 4 Interactions of the two IDs with the solenoid of RPK2-LRR. A, Detailed interactions of ID-1 with one lateral side of RPK2-LRR. The side chains from the ID-1 are shown in yellow and those from LRRs in purple. Red dashed lines represent hydrogen bonds. B, Detailed interactions of ID-2 with the inner surface side of RPK2-LRR. The side chains from the ID-2 are shown in cyan. C, Detailed interactions between ID-1 and ID-2.
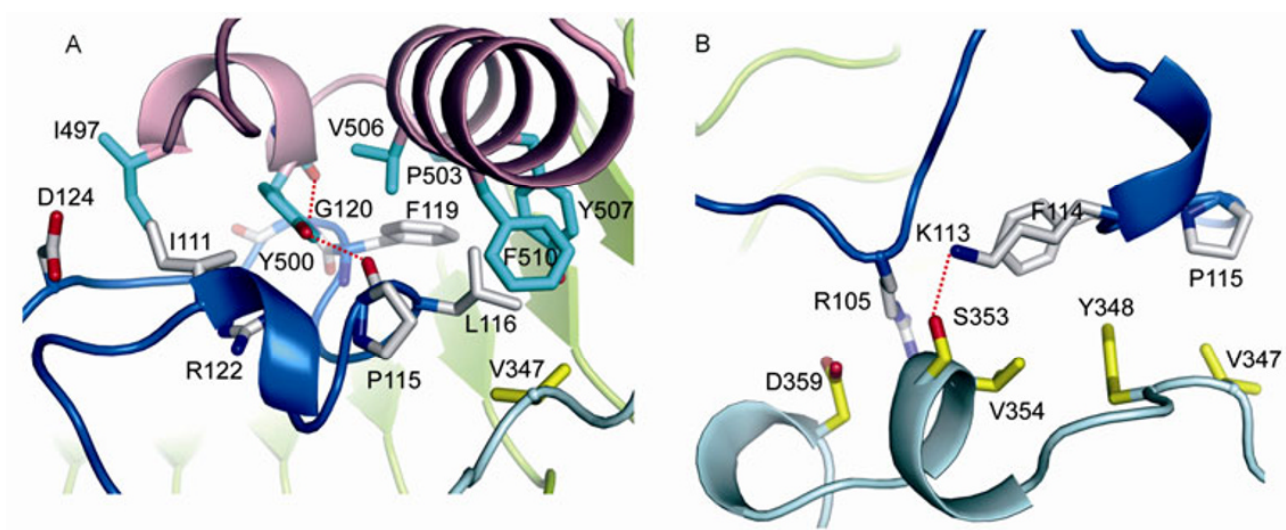

Figure 5 A long loop from the N-terminal side stabilizes the conformations of the two IDs. A, Detailed interactions of ID-1 with the long loop from the N-terminal side RPK2-LRR. The side chains from the ID-1 are shown in cyan and those from the loop in gray. Red dashed lines represent hydrogen bonds. B, Detailed interactions of ID-2 with the long loop from the N-terminal side RPK2-LRR. The side chains from the ID-2 are shown in yellow.

tions with the long loop are expected to further stabilize the conformations of the two IDs.

\subsection{A potential ligand binding site formed by the IDs and LRR solenoid}

Structural studies $[5,6]$ show that the hydrophobic groove between the ID and the LRR solenoid of BRI1-LRR acts as the BR (brassinosteroid) binding site at the C-terminal site. Intriguingly, in the structure of RPK2-LRR, a well defined pocket, close to the $\mathrm{C}$-terminal side, is formed by the two IDs and the inner surface of the LRR solenoid of RPK2LRR (Figure 6, left panel). The pocket is both negatively charged and hydrophobic (Figure 6, middle panel). In contrast with pocket-located side, the other side of the RPK2LRR solenoid is mainly positive on its surface (Figure 6, 

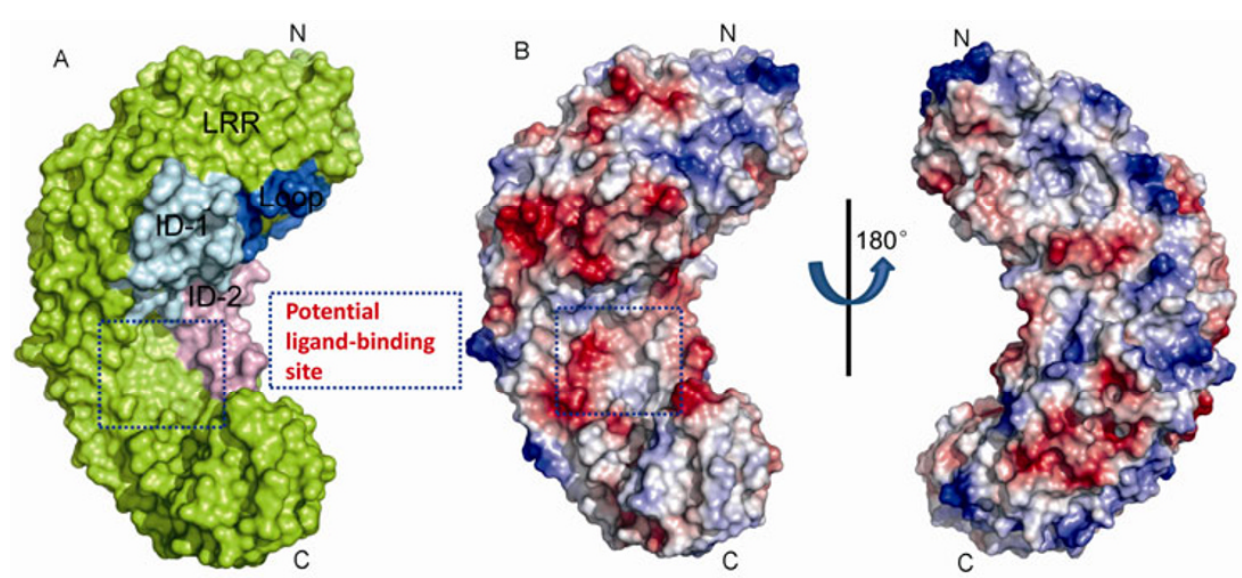

Figure 6 A, Potential ligand binding site formed by the IDs and LRR solenoid. B, Surface representations of RPK2-LRR. Colors for domains are the same as those shown in Figure 2A. The left and middle structures are shown in the same orientation. The pocket formed by the two IDs and the inner surface of LRR solenoid is highlighted in the squares and proposed to be a ligand binding site. " $\mathrm{N}$ " and " $\mathrm{C}$ " indicate the $\mathrm{N}$ - and C-terminus, respectively.

right panel). We propose that the pocket, similar to that observed in BRI1-LRR, may function as a site for binding ligand(s) of RPK2. Functional assays examining roles of the amino acids around this region in RPK2 signaling are needed for verifying validity of the hypothesis.

\section{Conclusion and perspectives}

Our crystal structure of RPK2-LRR reveals that its two IDs interact with the solenoid without disrupting the continuous packing of the LRRs, similar to what has been observed with BRI1-LRR [5,6] containing a single ID. The non-LRR region from BRI1-LRR has been shown to be important for ligand recognition $[5,6]$. Similarly, a surface groove created by interactions of the two IDs with the solenoid of RPK2-LRR could act as a scaffold for binding to its ligand(s). It is noteworthy that the two IDs are not conserved among different plant species (Figure 3). Thus, a question raised by our observation (Figure 6) is that whether RPK2s from these plants recognize a conserved ligand(s). A clear answer to this question awaits the identification of the RPK2's ligand(s).

We thank Huang Sheng and He JianHua at Shanghai Synchrotron Radiation Facility for assistance with data collection. This work was supported by the National Natural Science Foundation of China (31130063) and the National Outstanding Young Scholar Science Foundation of China (31025008) to Chai JiJie.

1 Kobe B, Kajava AV. The leucine-rich repeat as a protein recognition motif. Curr Opin Struct Biol, 2001, 11: 725-732

2 Botos I, Segal DM, Davies DR. The structural biology of Toll-like receptors. Structure, 2011, 19: 447-459

3 She J, Han Z, Zhou B, Chai J. Structural basis for differential recognition of brassinolide by its receptors. Protein Cell, 2013, 4: 475-482

4 Liu P, Hu Z, Zhou B, Liu S, Chai J. Crystal structure of an LRR protein with two solenoids. Cell Res, 2013, 23: 303-305
5 She J, Han Z, Kim TW, Wang J, Cheng W, Chang J, Shi S, Wang J, Yang M, Wang ZY, Chai J. Structural insight into brassinosteroid perception by BRI1. Nature, 2011, 474: 472-476

6 Hothorn M, Belkhadir Y, Dreux M, Dabi T, Noel JP, Wilson IA, Chory J. Structural basis of steroid hormone perception by the receptor kinase BRI1. Nature, 2011, 474: 467-471

7 Shiu SH, Bleecker AB. Plant receptor-like kinase gene family: diversity, function, and signaling. Science's STKE, 2001, 2001: re22

8 Matsushima N, Mikami T, Tanaka T, Miyashita H, Yamada K, Kuroki Y. Analyses of non-leucine-rich repeat (non-LRR) regions intervening between LRRs in proteins. Biochim Biophys Acta, 2009, 1790: 1217-1237

9 Schoof H, Lenhard M, Haecker A, Shinozaki K, Fukuda H, Sawa S. The stem cell population of Arabidopsis shoot meristems is maintained by a regulatory loop between the CLAVATA and WUSCHEL genes. Cell, 2000, 100: 635-644

10 Brand U, Fletcher JC, Hobe M, Meyerowitz EM, Simon R. Dependence of stem cell fate in Arabidopsis on a feedback loop regulated by CLV3 activity. Science, 2000, 289: 617-619

11 Stahl Y, Simon R. Plant primary meristems: shared functions and regulatory mechanisms. Curr Opin Plant Biol, 2010, 13: 53-58

12 Gao X, Guo Y. CLE peptides in plants: proteolytic processing, structure-activity relationship, and ligand-receptor interaction. J Integr Plant Biol, 2012, 54: 738-745

13 Miwa H, Kinoshita A, Fukuda H, Sawa S. Plant meristems: CLAVATA3/ESR-related signaling in the shoot apical meristem and the root apical meristem. J Plant Res, 2009, 122: 31-39

14 Kinoshita A, Betsuyaku S, Osakabe Y, Mizuno S, Nagawa S, Stahl Y, Simon R, Yamaguchi-Shinozaki K, Fukuda H, Sawa S. RPK2 is an essential receptor-like kinase that transmits the CLV3 signal in Arabidopsis. Development, 2010, 137: 3911-3920

15 Sawa S, Tabata R. RPK2 functions in diverged CLE signaling. Plant Signal Behav, 2011, 6: 86-88

16 Guo Y, Ni J, Denver R, Wang X, Clark SE. Mechanisms of molecular mimicry of plant CLE peptide ligands by the parasitic nematode. Plant Physiol, 2011, 157: 476-484

17 Replogle A, Wang J, Paolillo V, Smeda J, Kinoshita A, Durbak A, Tax FE, Wang X, Sawa S, Mitchum MG. Synergistic interaction of CLAVATA1, CLAVATA2, and RECEPTOR-LIKE PROTEIN KINASE 2 in cyst nematode parasitism of Arabidopsis. Mol Plant Microbe In, 2013, 26: 87-96

18 Nodine MD, Yadegari R, Tax FE. RPK1 and TOAD2 are two receptor-like kinases redundantly required for Arabidopsis embryonic pattern formation. Dev Cell, 2007, 12: 943-956

19 Nodine MD, Tax FE. Two receptor-like kinases required together for 
the establishment of Arabidopsis cotyledon primordia. Dev Biol, 2008, 314: 161-170

20 Mizuno S, Osakabe Y, Maruyama K, Ito T, Osakabe K, Sato T, Shinozaki K, Yamaguchi-Shinozaki K. Receptor-like protein kinase 2 (RPK2) is a novel factor controlling anther development in Arabidopsis thaliana. Plant J, 2007, 50: 751-766

21 Zhao D. Control of anther cell differentiation: a teamwork of receptor-like kinases. Sex Plant Reprod, 2009, 22: 221-228

22 Otwinowski Z, Minor W. Processing of X-ray diffraction data collected in oscillation mode. Method Enzymol, 1997, 276: 307-326

23 Collaborative Computational Project, Number 4. The CCP4 suite: programs for protein crystallography. Acta Crystallogr, 1994, D50: 760-763

24 Emsley P, Cowtan KC. Model-building tools for molecular graphics.
Acta Crystallogr D Biol Crystallogr, 2004, 60: 2126-2132

25 Adams P, Grosse KR, Huang LW, Ioerger TR, McCoy AJ, Moriarty NW, Read RJ, Sacchettini JC, Sauter NK, Terwilliger TC. PHENIX: building new software for automated crystallographic structure determination. Acta Crystallogr, 2002, D58: 1948-1954

26 DeLano WL. PyMOL molecular viewer. 2002, http://www.pymol. org/

27 Kim TW, Wang ZY. Brassinosteroid signal transduction from receptor kinases to transcription factors. Annu Rev Plant Biol, 2010, 61: 681-704

28 Sheard LB, Tan X, Mao H, Withers J, Ben-Nissan G, Hinds TR, Kobayashi Y, Hsu FF, Sharon M, Browse J, He SY, Rizo J, Howe GA, Zheng N. Jasmonate perception by inositol-phosphatepotentiated COI1-JAZ co-receptor. Nature, 2010, 468: 400-405

Open Access This article is distributed under the terms of the Creative Commons Attribution License which permits any use, distribution, and reproduction in any medium, provided the original author(s) and source are credited. 